Article

\title{
Screening and Distribution of Contaminants of Emerging Concern and Regulated Organic Pollutants in the Heavily Modified Guadalhorce River Basin, Southern Spain
}

\author{
Marta Llamas ${ }^{1}$, Iñaki Vadillo-Pérez ${ }^{1, *}$, Lucila Candela ${ }^{2} \mathbb{D}$, Pablo Jiménez-Gavilán ${ }^{1} \mathbb{D}_{\text {, }}$ \\ Carmen Corada-Fernández ${ }^{3}$ and Antonio F. Castro-Gámez ${ }^{1,4}$ \\ 1 Hydrogeology Group, Faculty of Science, University of Málaga, 29071 Málaga, Spain; \\ mllamas@uma.es (M.L.); pgavilan@uma.es (P.J.-G.); antoniof.castro@juntadeandalucia.es (A.F.C.-G.) \\ 2 IMDEA-Water, 28805 Alcalá de Henares, IUACA, 03690 Alicante, Spain; lucila.candela@imdea.org \\ 3 Physical Chemistry Department, Marine Research Institute, Campus de Excelencia Internacional del \\ Mar (CEIMAR), University of Cadiz, 11510 Cádiz, Spain; carmen.corada@uca.es \\ 4 General Office of Water Infrastructures, Department of Hydrology, 29016 Málaga, Spain \\ * Correspondence: vadillo@uma.es; Tel.: +34-95-213-42-13
}

Received: 1 September 2020; Accepted: 22 October 2020; Published: 27 October 2020

\begin{abstract}
Emerging pollutants have aroused an increasing concern due to their ubiquitous presence in the environment and harmful potential. Both emerging (e.g., pharmaceuticals and personal care products) and regulated organic pollutants pose a serious threat to water quality and their presence and spatial distribution are complicated to address as they can derive from several factors: distribution of point and diffuse sources, environmental conditions, hydrogeological features of the region and inherent properties of the considered contaminants. In this study, a ground and surface water monitoring campaign was conducted in the three main detritic groundwater bodies of an extensive and heavily modified river basin in order to draft an initial description of the occurrence and distribution of a wide range of organic contaminants. In total, 63 out of 185 target pollutants were detected. An attempt to understand the importance of different factors governing the distribution of some of the most frequently found pollutants was made. Antibiotics spatial distribution is potentially influenced by the hydrogeological functioning of the basin modified by hydraulic infrastructures (reflected by hydrochemistry and environmental tracers $\delta^{2} \mathrm{H}$ and $\delta^{18} \mathrm{O}$ ), not directly related to the distribution of potential sources. The presence of other organic pollutants does not reflect an evident correlation with flow pathways. Differences in contaminant occurrence are potentially attributed to the way pollutants are released into the environment as well as physico-chemical properties.
\end{abstract}

Keywords: emerging contaminants; organic contaminants; groundwater; environmental tracers; large scale

\section{Introduction}

Society today uses a broad array of synthetic organic compounds on a daily basis for purposes like household activities, industrial manufacturing, agricultural applications and human and animal healthcare. These chemical products as well as other organic compounds derived from anthropogenic activities have reached the terrestrial and aquatic environment, sparking a growing interest and concern about their environmental fate and toxicity [1]. Among these, pharmaceutically active compounds (PhACs) and personal care products (PCPs) are regarded as contaminants of emerging concern, many of them with unknown geochemical behavior, which may trigger undesirable effects 
on water resource availability in the long term. Moreover, their presence in the environment is largely unregulated by legislation. Currently, threshold values are established only for nitrates and pesticides for groundwater [2]. Nonetheless, some PhACs and PCPs have already been placed on a "Watch List" [3], so their presence in the aquatic environment could eventually be contemplated by EU legislation.

PCPs are a very important group of pollutants in terms of production volume. It includes fragrances, UV filters, insect repellents, antibacterials, surfactants and some new flame retardants, among others. Emerging pollutants do not need to be highly persistent to negatively affect ecosystems and human health [4-6] as their degradation is offset by a continuous input into the environment $[7,8]$.

Emerging and regulated (e.g., pesticides and polycyclic aromatic hydrocarbons) organic contaminants enter groundwater from several sources, such as treated [9] or untreated domestic and industrial effluents, leachate from landfills, leakages in septic tanks and sewerage, agricultural practices (i.e., use of pesticides and irrigation with reclaimed wastewater [10]), livestock activities (i.e., waste lagoons and manure application to soil; [11]) and also indirectly through groundwater-surface water exchange processes [1]. In addition, special conditions such as the combination of extreme rainfall events and raw wastewater can cause a sewer overflow, resulting in significant loads of organic contaminants in some areas [12].

Once released into the environment, natural attenuation of organic pollutants depends on several processes such as dilution, hydrolysis, biodegradation and sorption, among others. Sorption processes in the subsurface depends on both their physicochemical properties and environmental factors [13]. The fate of non-ionizable compounds is determined by hydrophobic sorption, whereas the charge state of ionizable molecules can influence several key characteristics (e.g., volatility, hydrophilicity, reactivity, sorption affinity; [14]), which makes $\mathrm{pH}$ an important environmental condition. Other environmental factors playing an important role in organic pollutant attenuation are temperature and redox potential [15], which can also promote or limit biodegradation [11].

Consequently, a very uneven distribution of regulated and non-regulated organic pollutants in groundwater is expected at a large scale given the wide variety of factors involved: distribution of potential sources in the area, environmental factors, hydrogeological features of the region and physico-chemical characteristics of the considered compounds [16].

In this research, the presence of contaminants of emerging concern and regulated organic pollutants in groundwater in an extensive and highly modified river basin is assessed with the aim to provide an initial description of the area regarding water pollution by organic chemicals. To this end, a surface and groundwater monitoring campaign was carried out in detritic aquifers to investigate the spatial distribution and occurrence of 185 microcontaminants of different groups of classes: polychlorinated biphenyls (PCBs), polycyclic aromatic hydrocarbons (PAHs) and pesticides; and emerging pollutants: PhACs, PCPs and organophosphorus flame retardants (OPFRs). Further research will be optimized based on the obtained results.

\section{Study Area}

The Guadalhorce River basin is located in southern Spain. It extends over an area of approximately $3200 \mathrm{~km}^{2}$ and the length of the river is $166 \mathrm{~km}$. As shown in Figure 1, the river is born in the northeast of the basin and flows into the Mediterranean Sea to the southwest of the city of Malaga. The climate characteristics of the area are mild temperatures, whose annual mean value varies from $13{ }^{\circ} \mathrm{C}$ to $18{ }^{\circ} \mathrm{C}$, and a defined precipitation regime throughout the year: October to February are the wettest seasons when extreme events occur frequently, with summer (June to September) being the driest season. Rainfall values range from 400 to $500 \mathrm{~mm} /$ year in the southern part of the basin and from 900 to $1000 \mathrm{~mm} /$ year in the northern part [17]. 


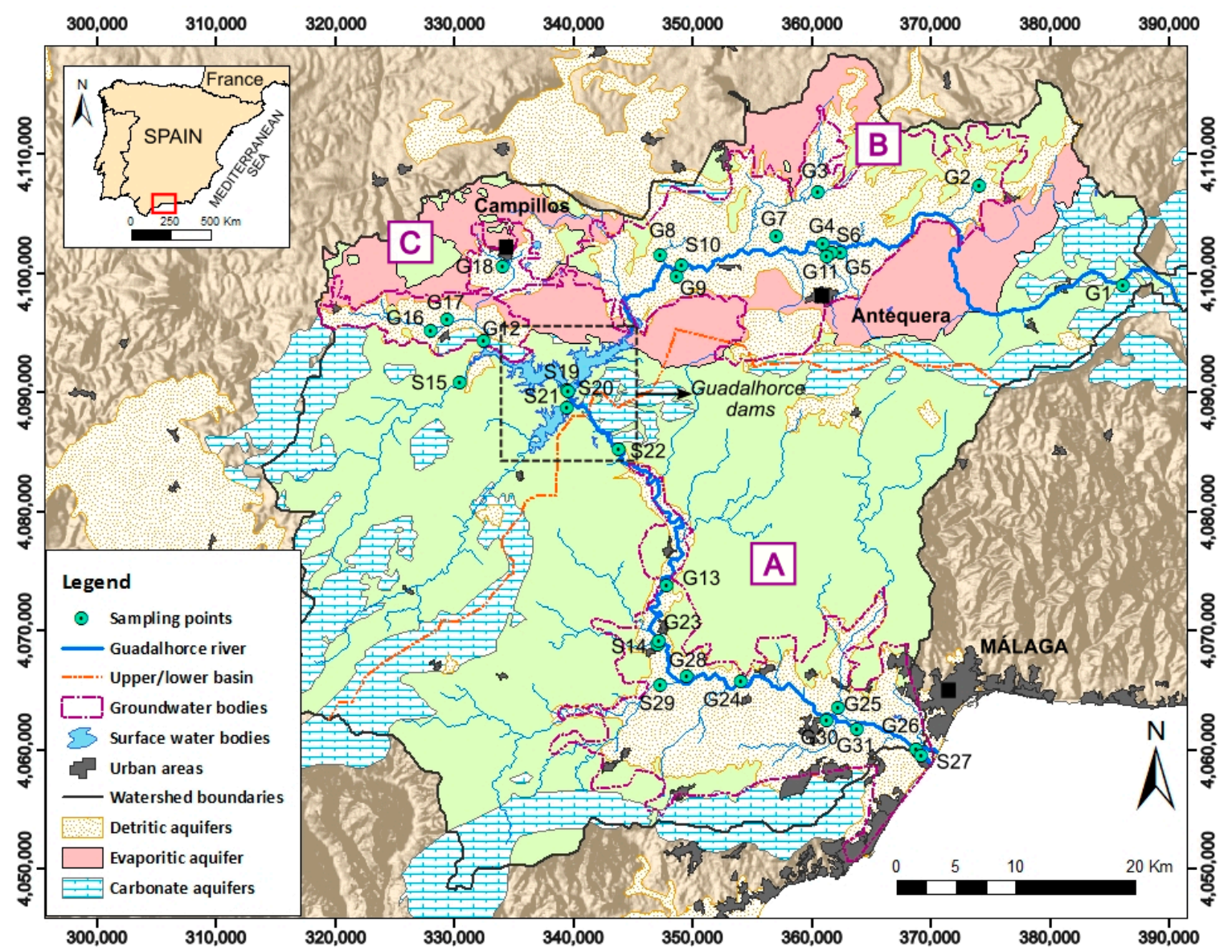

Figure 1. The Guadalhorce River basin and the three target areas (A, B and C) with the sampling points.

The Guadalhorce river basin can be divided into two sectors due to the presence of a mountain range located in the center: the northern sub-basin whose flatlands are located at a higher altitude (between 300 and $600 \mathrm{~m}$ a.s.l.), and the southern sub-basin (between 0 and $200 \mathrm{~m}$ a.s.l. given the altitude of its flatlands) [17]. This division is also based on the hydrological functioning of the two sub-basins, which is influenced by the presence of three dams that collect water from the entire upper basin (Figure 1).

Three aquifer types have been identified in the basin: carbonate, porous and evaporitic [17]. Carbonate aquifers, whose springs feed the main surface watercourses, are formed by Mesozoic limestones, dolostones and marbles of the external and internal zones of the Betic Cordillera. The Malaguide Complex, a tectonic unit included in the internal zone, is on the right bank of the Guadalhorce River, near the city of Malaga, and contains greywackes and phyllites (shales) with disseminated pyrite and organic matter [18].

The Triassic clay, sandstone and evaporative material (gypsum and halite) outcrops [17] form the only evaporitic aquifer, which is in the northern sub-basin. These materials also constitute the basement of the porous and carbonate aquifers of the basin.

The target areas of this work are three porous aquifers located in the flatlands of the basin: the Lower Guadalhorce (sector A; Figure 1), situated in the lower part of the basin, and Vega de Antequera-Archidona (sector B; Figure 1) and the Teba-Almargen-Campillos area (sector C; Figure 1) situated in the upper sub-basin.

In sector A (Lower Guadalhorce), the Quaternary and unconfined aquifer is formed by alluvial sediments such as gravels, sands, silts and clays. The underlying rocks are Upper Miocene calcareous sandstones and conglomerates, and Pliocene conglomerates, marl and sand layers. Pliocene sediments can be $300 \mathrm{~m}$ thick and, at the bottom of the series, conglomerates form a discontinuous confined 
aquifer underlying the marls. At a shallower level, interrupted sand layers act as a semiconfined aquifer (Figure 2 [19-21]).

The Vega de Antequera-Archidona aquifer (sector B) consists of Neogene and Quaternary deposits such as calcareous sandstones and alluvial sediments [22].

The Teba-Almargen-Campillos (sector C) system is formed by two detritic aquifers and one carbonate aquifer that are hydrologically connected. Calcarenites, conglomerates and marls (Miocene) and detrital materials of fluvial origin (Quaternary) constitute the detritic aquifers and Jurassic limestones form the carbonate one [23].

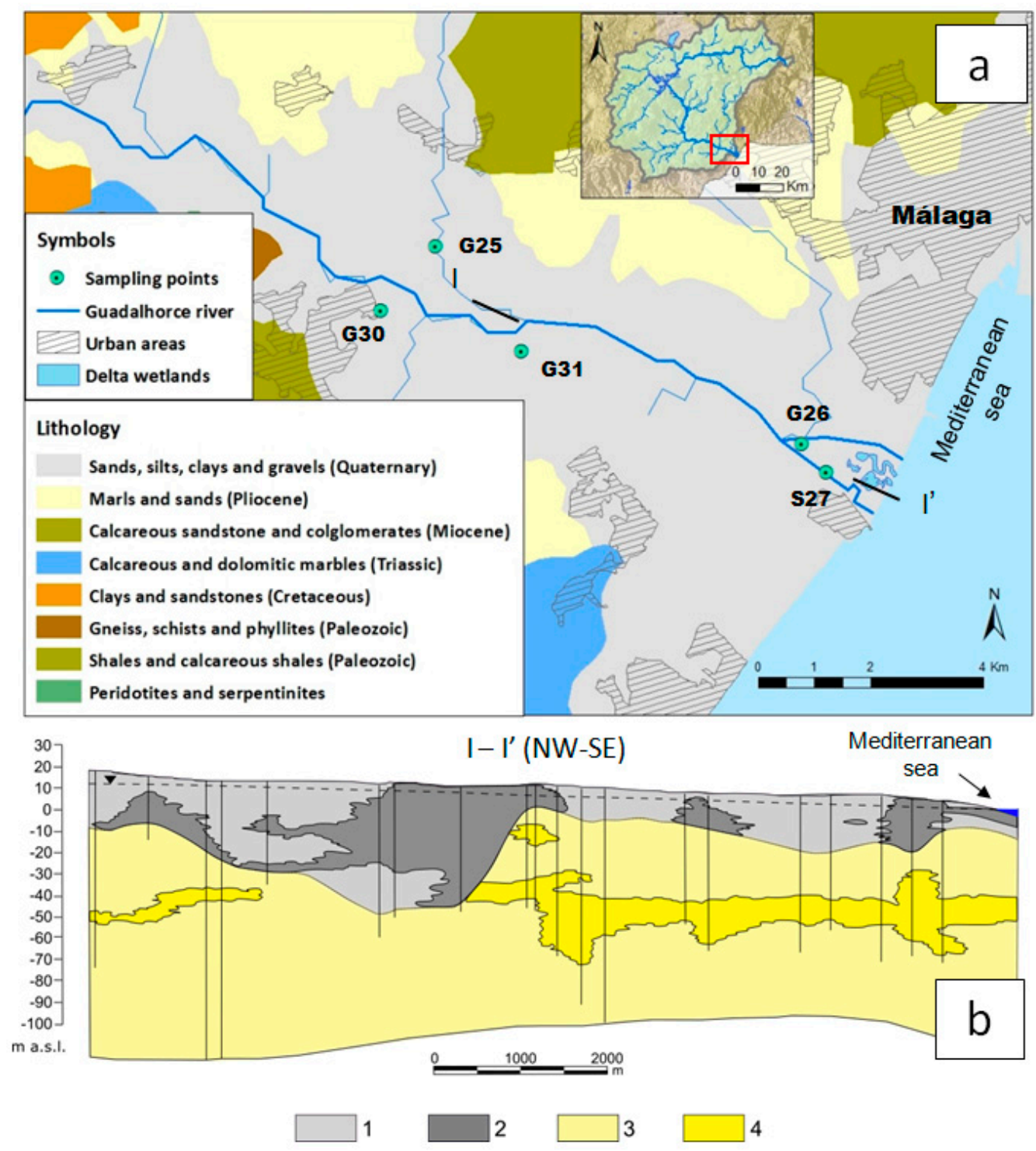

Figure 2. Geological scheme of sector A (a) and hydrogeological section (b) (modified from [19-21]). Legend: 1: Quaternary aquifer formed predominantly by gravels and sands; 2: Quaternary aquifer consisting of sands, silts and clays; 3: low-permeability Pliocene clays and marls; 4: semiconfined Pliocene aquifer formed by gravels and sands.

\section{Materials and Methods}

\subsection{Water Sample Collection and Preparation}

In the March 2016 sampling campaign, 31 groundwater and surface water samples (Figure 1) were collected. Groundwater samples (21) corresponded to the porous aquifers and were collected 
directly from the wells ( $<30-40 \mathrm{~m}$ deep) after continuous pumping; sample G26 was the only one collected using a submersible sampler. All water samples were filtered through a $0.45 \mu \mathrm{m}$ Millipore ${ }^{\circledR}$ (Merck KGaA, Darmstadt, Germany) filter. Samples for hydrochemical and isotopic analyses were stored in sterile high-density polyethylene bottles $(120 \mathrm{~mL})$ sealed with inverted cone caps; sterile amber glass bottles $(1 \mathrm{~L})$ with Teflon caps were used for samples to be analyzed for organic compounds. All bottles were rinsed before sampling, carried in a cool-box and then stored in a fridge below $4{ }^{\circ} \mathrm{C}$ until analysis was performed, generally within $24 \mathrm{~h}$ of sampling.

In situ physico-chemical parameters: $\mathrm{pH}$, temperature, electrical conductivity (EC), redox potential (Eh) and dissolved oxygen (DO) were measured with a portable multi-parameter probe Hach-Lange HQ40d (Hach, Loveland, CO, USA) and a flow cell to avoid contact with the atmosphere (Table 1). Electrical conductivity was calibrated with a $\mathrm{NaCl}$ standard solution. The $\mathrm{pH}$ was calibrated with 4 and $7 \mathrm{pH}$ buffer solutions.

Table 1. Descriptive statistics for physicochemical parameters of monitored groundwater in sectors A, $B$ and $C$.

\begin{tabular}{cccccc}
\hline & pH & EC $(\mu \mathrm{S} / \mathbf{c m})$ & Temperature $\left({ }^{\circ} \mathbf{C}\right)$ & Eh $(\mathbf{m V})$ & $\mathbf{O}_{\mathbf{2}}(\mathbf{m g} / \mathbf{L})$ \\
\hline & \multicolumn{5}{c}{ Sector A } \\
\hline Min. & 7.0 & 1877 & 16.2 & 105 & 3.4 \\
Max. & 9.1 & 4100 & 19.0 & 203 & 7.6 \\
Median & 7.4 & 2280 & 18.3 & 190 & 5.0 \\
Mean & 7.5 & 2640 & 18.0 & 181 & 5.5 \\
Std. dev. & 0.6 & 777 & 1.0 & 32 & 1.7 \\
\hline & & & Sector B & & \\
\hline Min. & 7.0 & 305 & 14.2 & 187 & 7.0 \\
Max. & 7.7 & 2300 & 18.0 & 277 & 8.9 \\
Median & 7.1 & 1626 & 15.0 & 241 & 8.8 \\
Mean & 7.2 & 1518 & 15.5 & 235 & 8.2 \\
Std. dev. & 0.2 & 631 & 1.2 & 27 & 0.8 \\
\hline & & & Sector C & \\
\hline Min. & 7.1 & 495 & 11.6 & 160 & 3.2 \\
Max. & 8.3 & 3730 & 18.5 & 235 & 9.8 \\
Median & 7.4 & 1454 & 15.2 & 198 & 5.9 \\
Mean & 7.5 & 1775 & 15.2 & 201 & 6.0 \\
Std. dev. & 0.4 & 1192 & 2.4 & 28 & 2.7 \\
\hline
\end{tabular}

\subsection{Hydrochemical and Isotopic Analysis}

Hydrochemical analysis (Table 2) consisted in the determination of major and minor components $\left(\mathrm{Ca}^{2+}, \mathrm{Mg}^{2+}, \mathrm{Na}^{+}, \mathrm{K}^{+}, \mathrm{HCO}_{3}{ }^{-}, \mathrm{Cl}^{-}, \mathrm{SO}_{4}{ }^{2-}\right.$ and $\left.\mathrm{NO}_{3}{ }^{-}\right)$and it was performed using HPLC with a Metrohm 881 Compact IC Pro. $\delta^{2} \mathrm{H}$ and $\delta^{18} \mathrm{O}$ were determined with laser spectroscopy (CRDS) in a Picarro Water Isotope Analyzer L2120i. Notation for $\delta^{2} \mathrm{H}$ and $\delta^{18} \mathrm{O}$ is expressed in \%o in relation to the international standard Vienna Standard Mean Oceanic Water (V-SMOW). Hydrochemical and isotope determinations were performed at the laboratory of the Center of Hydrogeology of the University of Malaga. 
Table 2. Descriptive statistics for hydrochemical parameters and stable isotopes $\delta^{2} \mathrm{H}$ and $\delta^{18} \mathrm{O}$ of sectors $\mathrm{A}, \mathrm{B}$ and $\mathrm{C}$.

\begin{tabular}{|c|c|c|c|c|c|c|c|c|c|c|}
\hline & $\begin{array}{c}\mathrm{Ca}^{2+} \\
(\mathrm{mg} / \mathrm{L})\end{array}$ & $\begin{array}{c}\mathrm{Mg}^{2+} \\
(\mathrm{mg} / \mathrm{L})\end{array}$ & $\begin{array}{c}\mathrm{Na}^{+} \\
(\mathrm{mg} / \mathrm{L})\end{array}$ & $\begin{array}{c}\mathrm{K}^{+} \\
(\mathrm{mg} / \mathrm{L})\end{array}$ & $\begin{array}{l}\mathrm{HCO}_{3}{ }^{-} \\
(\mathrm{mg} / \mathrm{L})\end{array}$ & $\begin{array}{l}\mathrm{SO}_{4}^{2-} \\
(\mathrm{mg} / \mathrm{L})\end{array}$ & $\begin{array}{c}\mathrm{Cl}^{-} \\
(\mathrm{mg} / \mathrm{L})\end{array}$ & $\begin{array}{l}\mathrm{NO}_{3}^{-} \\
(\mathrm{mg} /)\end{array}$ & $\begin{array}{c}\delta^{2} \mathrm{H} \\
(\% o)\end{array}$ & $\begin{array}{l}\delta^{18} \mathrm{O} \\
(\% o)\end{array}$ \\
\hline \multicolumn{11}{|c|}{ Sector A } \\
\hline Min. & 7.4 & 0.8 & 5.6 & 0.4 & 96.2 & 66.7 & 123.1 & 2.9 & -31.8 & -5.1 \\
\hline Max. & 283.8 & 138.9 & 526.3 & 7.6 & 474.4 & 233.5 & 634.3 & 58.5 & -28.2 & -4.0 \\
\hline Median & 115.5 & 80.7 & 207.5 & 3.9 & 371.7 & 186.2 & 234.1 & 18.9 & -30.2 & -4.8 \\
\hline Mean & 112.2 & 81.8 & 243.3 & 3.7 & 353.7 & 169.3 & 273.7 & 25.0 & -30.0 & -4.6 \\
\hline Std. dev. & 95.3 & 44.9 & 189.3 & 2.5 & 112.8 & 55.3 & 164.6 & 20.4 & 1.4 & 0.4 \\
\hline \multicolumn{11}{|c|}{ Sector B } \\
\hline Min. & 54.3 & 6.3 & 1.8 & 0.3 & 191.1 & 8.5 & 3.6 & 3.8 & -43.5 & -7.6 \\
\hline Max. & 316.0 & 82.2 & 165.9 & 3.7 & 301.0 & 2007.1 & 197.5 & 155.9 & -34.8 & -5.0 \\
\hline Median & 244.2 & 50.3 & 58.6 & 2.3 & 270.1 & 291.2 & 81.2 & 75.6 & -37.5 & -5.8 \\
\hline Mean & 208.3 & 48.6 & 67.2 & 2.3 & 260.7 & 786.9 & 82.4 & 70.0 & -37.8 & -5.9 \\
\hline Std. dev. & 99.2 & 26.7 & 50.7 & 1.0 & 40.9 & 823.2 & 62.0 & 44.8 & 2.5 & 0.7 \\
\hline \multicolumn{11}{|c|}{ Sector C } \\
\hline Min. & 57.6 & 10.6 & 13.6 & 1.6 & 243.7 & 20.3 & 10.4 & 5.5 & -41.4 & -6.5 \\
\hline Max. & 381.9 & 136.7 & 306.0 & 4.8 & 340.8 & 664.2 & 300.1 & 433.8 & -33.3 & -5.2 \\
\hline Median & 135.7 & 45.4 & 120.1 & 2.6 & 284.5 & 143.5 & 88.1 & 44.8 & -36.5 & -5.6 \\
\hline Mean & 160.6 & 57.8 & 130.2 & 2.8 & 285.5 & 242.3 & 105.9 & 108.4 & -36.8 & -5.8 \\
\hline Std. dev. & 129.0 & 52.2 & 113.8 & 1.3 & 35.7 & 265.3 & 113.9 & 183.2 & 3.4 & 0.6 \\
\hline
\end{tabular}

\subsection{Analysis of Emerging and Regulated Compounds}

Chemical analysis on water samples of 185 microcontaminants was performed; selected target compounds are listed in Table 3. Analysis of pharmaceutically active compounds (PhACs) was performed by solid-phase extraction (SPE) followed by ultra-performance liquid chromatography-triple quadrupole mass spectrometry (UPLC-QqQ-MS/MS) using a Bruker EVOQ Elite system (Bruker, Billerica, MA, USA) equipped with an electrospray ionization source [24].

For personal care products (PCPs), polycyclic aromatic hydrocarbons (PAHs), polychlorinated biphenyls (PCBs), organophosphorus components used as flame retardants (OPFRs) and several types of pesticides (organochlorine and organophosphorus pesticides, triazines and pyrethroids), samples were processed using stir bar sorptive extraction (SBSE) [25]. Separation, identification and quantification of target contaminants were performed using gas chromatography (SCION 456-GC, Bruker) coupled to triple quadrupole mass spectrometry (SCION TQ from Bruker with CP 8400 Autosampler) [26]. Analyses were conducted in the laboratory of the Marine Research Institute of the University of Cadiz.

Further information on the limits of detection, extraction recoveries and performance of the analytical methods used here can be found in the aforementioned references [24-26].

\section{Results and Discussion}

\subsection{Water Chemistry and Hydrochemical Water Types}

For all analyzed samples, $\mathrm{pH}$ values ranged from 7.0 to slightly alkaline (maximum $\mathrm{pH}$ of 9.1 in sector A). Eh maximum values are lower than $300 \mathrm{mV}$, reflecting weak anoxic conditions which are also revealed by low mean values of dissolved oxygen in sectors A and C. A summary of descriptive statistics for physico-chemical and hydrochemical parameters recorded in groundwater is presented in Tables 1 and 2 .

Sectors A, B and C revealed EC values of up to 4100,2300 and $3730 \mu \mathrm{S} / \mathrm{cm}$, respectively, showing a high mineralization in some sampling points. The EC mean value in sector A can be explained by anthropogenic pollution sources and seawater influence in areas close to the sea border. High concentrations of sulfate in sector B (Table 2) constitute an important contribution to groundwater 
mineralization in this sector. The basement of the porous aquifers, partly constituted by evaporitic materials (Triassic), is responsible for high natural background levels of dissolved $\mathrm{SO}_{4}{ }^{2-}$, especially in the upper basin (sectors B and C). However, in the lower basin, the high concentrations of dissolved $\mathrm{SO}_{4}{ }^{2-}$ are essentially a consequence of the use of fertilizers, which represents over $80 \%$ of the total [27].

The Piper diagram (Figure 3) reveals the occurrence of four water types or hydrochemical facies of the monitored groundwater: $\mathrm{HCO}_{3}{ }^{-}-\mathrm{Ca}^{2+}, \mathrm{SO}_{4}{ }^{2-}-\mathrm{Ca}^{2+}, \mathrm{Cl}^{-}-\mathrm{Na}^{+}$and mixed types. Most samples from sector $\mathrm{B}$ reveal $\mathrm{SO}_{4}{ }^{2-}-\mathrm{Ca}^{2+}$ facies, reflecting the influence of the evaporitic materials of the basement. Sampling points $\mathrm{G} 1$ and $\mathrm{G} 12\left(\mathrm{HCO}_{3}{ }^{-}-\mathrm{Ca}^{2+}\right)$ drain carbonate materials. The chemical composition of $\mathrm{G} 26$ $\left(\mathrm{Cl}-\mathrm{Na}^{+}\right)$, situated near the mouth of the Guadalhorce River, suggests seawater influence. Mixed types are essentially represented by water samples from the lower sub-basin (sector A), which means there are several processes or water sources involved in the formation of this water composition.

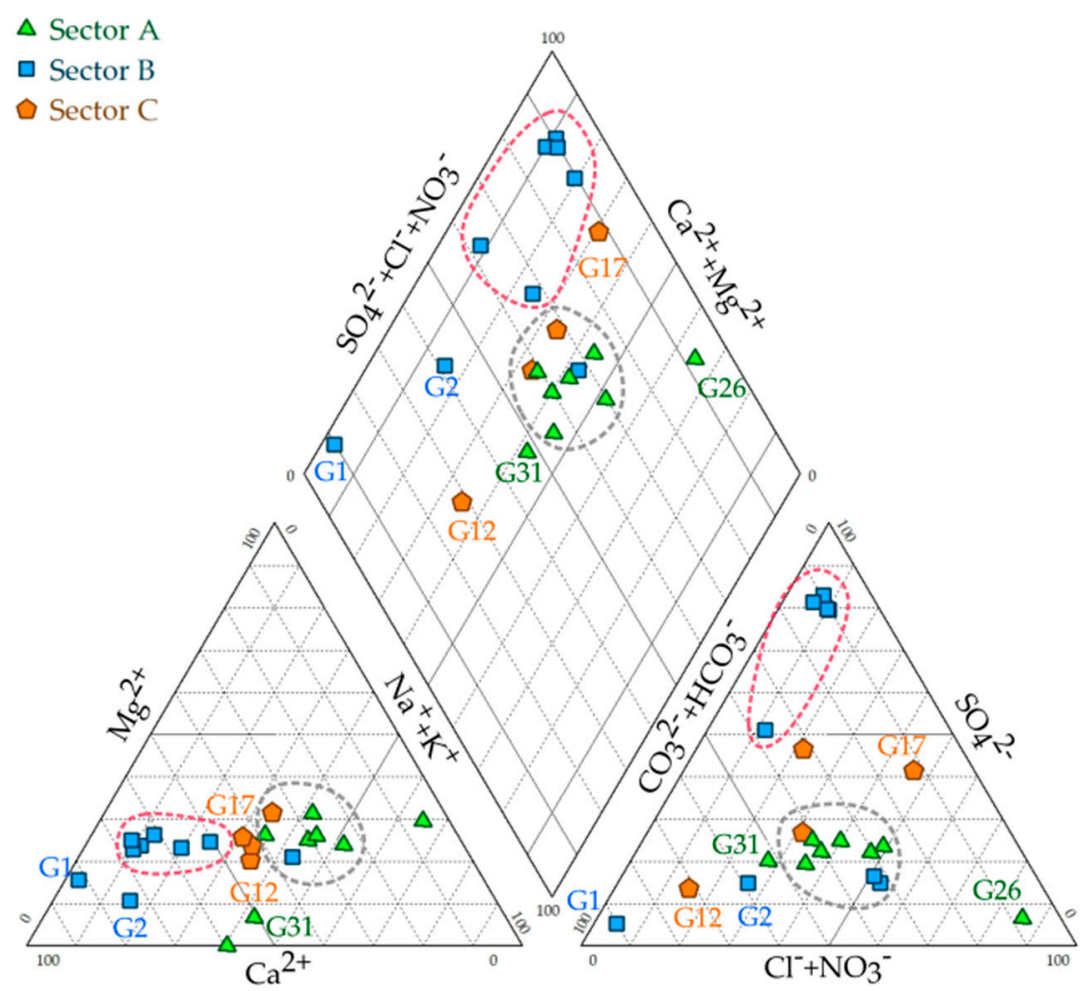

Figure 3. Piper diagram showing hydrochemical facies of monitored groundwater from sectors A, B and $\mathrm{C}$.

\subsection{Organic Active Compounds}

In total, 63 out of 185 target pollutants were detected in the study area (Table 3), of which 21 were found at least in $25 \%$ of the groundwater samples (Figure 4 ). On average, 17 compounds were detected per groundwater sampling point. The maximum number of contaminants was observed in G26 (55 compounds) located in sector A, near the mouth of the Guadalhorce River. The high presence of compounds is potentially due to a waste water treatment plant (WWTP, near Malaga City) located upstream and possible discharges from an existing industrial site. 
Table 3. Analyzed compounds, CAS (Chemical Abstracts Service) number and use. Legend: PCPs: personal care products; OPFRs: organophosphorus flame retardants; PAHs: polycyclic aromatic hydrocarbons; PCBs: polychlorinated biphenyls. Detected compounds are indicated in bold type.

\begin{tabular}{|c|c|c|c|c|c|c|c|c|}
\hline \multicolumn{9}{|c|}{ Compounds } \\
\hline Pharmaceuticals & CAS & Use & PCPs & CAS & Use & Pesticides & CAS & Use \\
\hline $17 \alpha$-ethynylestradiol ab & $57-63-6$ & Estrogen & OD-PABA & $21245-02-3$ & UV filter & Aldrin ${ }^{a}$ & $309-00-2$ & Organochlorine pesticide \\
\hline $17-\beta$ estradiol ab & $50-28-2$ & Estrogen & 2-OHBP & $117-99-7$ & UV filter & Ametryn & $834-12-8$ & Triazine \\
\hline Acetaminophen & $103-90-2$ & Analgesic/anti-inflammatory & 3-ОНРВ & $13020-57-0$ & UV filter & Atraton & $1610-17-9$ & Triazine \\
\hline Albuterol & $18559-94-9$ & Other PhACs & 4-OHPB & $1137-42-4$ & UV filter & Atrazineab & 1912-24-9 & Triazine \\
\hline Amitriptyline & $50-48-6$ & Psychiatric drug and stimulant & $4 \mathrm{MBC}$ & $36861-47-9$ & UV filter & Bifenthrin & $82657-04-3$ & Pyrethroid \\
\hline Amoxicillin & 26787-78-0 & Penicillin (antibiotic) & Benzyl salicylate & $118-58-1$ & UV filter & Carbophenothion & $786-19-6$ & Organophosphate pesticide \\
\hline Ampicillin & $69-53-4$ & Penicillin (antibiotic) & Bisphenol Ab & $80-05-7$ & Plasticizer & Chlorpyrifos ab & 2921-88-2 & Organophosphate pesticide \\
\hline Atenolol & 29122-68-7 & $\beta$-blocker (antihypertensive) & Cashmeran & $33704-61-9$ & Other fragrances & Cyfluthrin (I-IV) & $68359-37-5$ & Pyrethroid \\
\hline Atorvastatin & $134523-00-5$ & Lipid regulator & Celestolide & $13171-00-1$ & Polycyclic musk & $\begin{array}{l}\text { Cypermethrin } \\
\text { (I-IV) }\end{array}$ & $52315-07-8$ & Pyrethroid \\
\hline Azithromycin & $83905-01-5$ & Macrolide & DEET & $134-62-3$ & Insect repellent & Deltamethrin I,II & $52918-63-5$ & Pyrethroid \\
\hline Bezafibrate & $41859-67-0$ & Lipid regulator & EHMC & $83834-59-7$ & UV filter & Dieldrin $^{a}$ & $60-57-1$ & Organochlorine pesticide \\
\hline Caffeine & $58-08-2$ & Psychiatric drug and stimulant & Ethylhexyl salicylate & $118-60-5$ & UV filter & $\begin{array}{l}\text { Endosulfan } \\
\text { Sulfate }^{a}\end{array}$ & $1031-07-8$ & Organochlorine pesticide \\
\hline Carbamazepine & $298-46-4$ & Psychiatric drugs and stimulants & Exaltenone & $14595-54-1$ & Macrocyclic musk & Endrina & $72-20-8$ & Organochlorine pesticide \\
\hline Cefaclor & 53994-73-3 & Cephalosporin & Galaxolide & $1222-05-5$ & Polycyclic musks & Endrin Ketone & $53494-70-5$ & Organochlorine pesticide \\
\hline Cefadroxil & $50370-12-2$ & Cephalosporin & Habanolide & $34902-57-3$ & Macrocyclic musk & Ethion & $563-12-2$ & Organophosphate pesticide \\
\hline Cefdinir & $91832-40-5$ & Cephalosporin & Helvetolide & 141773-73-1 & Other fragrances & Fenvalerate I,II & 51630-58-1 & Pyrethroid \\
\hline Cefquinome & $84957-30-2$ & Cephalosporin & Homosalate & $118-56-9$ & UV filter & Heptachlor & $76-44-8$ & Organochlorine pesticide \\
\hline Ceftiofur & $80370-57-6$ & Cephalosporin & IRGAROL & 28159-98-0 & Insect repellent & $\begin{array}{l}\text { Heptachlor } \\
\text { Epoxide Isomer B }\end{array}$ & $1024-57-3$ & Organochlorine pesticide \\
\hline Chloramphenicol & $56-75-7$ & Amphenicol & Mexenone & $1641-17-4$ & UV filter & Lindane $^{b}$ & $58-89-9$ & Organochlorine pesticide \\
\hline Chlortetracycline & $57-62-5$ & Tetracyclines & MTCS $^{\text {b }}$ & $4640-01-1$ & Antibacterial & Metoxychlor & $72-43-5$ & Organochlorine pesticide \\
\hline Ciprofloxacin & 85721-33-1 & Quinolone (antibiotic) & Muscenone & 63314-79-4 & Macrocyclic musk & $o, p^{\prime}-D D T a b$ & 789-02-6 & Organochlorine pesticide \\
\hline Clarithromycin & 81103-11-9 & Macrolide & Muscone & $541-91-3$ & Macrocyclic musk & $\mathrm{p}, \mathbf{p}^{\prime}$-DDT $\mathrm{ab}$ & $72-54-8$ & Organochlorine pesticide \\
\hline Clindamycin & 18323-44-9 & Lincosamides (antibiotics) & Musk ambrette & $83-66-9$ & Nitro musk & $\mathrm{p}, \mathrm{p}^{\prime}-\mathrm{DDE} \mathrm{a}^{\mathrm{a}}$ & $72-55-9$ & Organochlorine pesticide \\
\hline
\end{tabular}


Table 3. Cont

\begin{tabular}{|c|c|c|c|c|c|c|c|c|}
\hline \multicolumn{9}{|c|}{ Compounds } \\
\hline Pharmaceuticals & CAS & Use & PCPs & CAS & Use & Pesticides & CAS & Use \\
\hline Clofibric acid & $882-09-7$ & Lipid regulator & Musk ketone & $81-14-1$ & Nitro musks & Parathion & $56-38-2$ & Organophosphate pesticide \\
\hline Danofloxacin & $112398-08-0$ & Quinolone (antibiotic) & Musk moskene & $116-66-5$ & Nitro musk & Permethrin I,II & 52645-53-1 & Pyrethroid \\
\hline Diclofenac ${ }^{b}$ & $15307-86-5$ & Analgesic/anti-inflammatory & Musk R1 & 3391-83-1 & Macrocyclic musk & Phenothrin I,II & $26002-80-2$ & Pyrethroid \\
\hline Doxycicline & $564-25-0$ & Tetracyclines & Musk tibetene & $145-39-1$ & Nitro musk & Prometon & $1610-18-0$ & Triazine \\
\hline Enrofloxacin & 93106-60-6 & Quinolone (antibiotic) & Musk xylene & $81-15-2$ & Nitro musk & Prometryn & 7287-19-6 & Triazine \\
\hline Erythromycin & $114-07-8$ & Macrolide & Nonylphenol $^{\mathrm{ab}}$ & 84852-15-3 & Plasticizer & Propazine & $139-40-2$ & Triazine \\
\hline Estrone $^{\mathrm{b}}$ & $53-16-7$ & Estrogen & Octylphenol ${ }^{\mathrm{ab}}$ & $1806-26-4$ & Plastizicer & Secbumeton & $26259-45-0$ & Triazine \\
\hline Famotidine & $76824-35-6$ & $\begin{array}{l}\text { Histamine } \mathrm{H} 2 / \text { receptor } \\
\text { antagonists }\end{array}$ & OTNE & $54464-57-2$ & Other fragrances & Simazine $^{\mathrm{a}}$ & $122-34-9$ & Triazine \\
\hline Fenofibrate & $49562-28-9$ & Lipid regulator & Oxybenzone & $131-57-7$ & UV filter & Simetryn & $1014-70-6$ & Triazine \\
\hline Fenoprofen & 29679-58-1 & Analgesic/anti-inflammatory & Phantolide & $15323-35-0$ & Polycyclic musk & Terbuthylazine & $5915-41-3$ & Triazine \\
\hline Flumequine & $42835-25-6$ & Quinolone (antibiotic) & Tonalide $\mathrm{b}$ & $1506-02-1$ & Polycyclic musks & Terbutryn ${ }^{a}$ & $886-50-0$ & Triazine \\
\hline Fluoxetine & $54910-89-3$ & Psychiatric drug and stimulant & Traseolide & $6814-48-7$ & Polycyclic musk & $\alpha$-chlordane & $5103-71-9$ & Organochlorine pesticide \\
\hline Furosemide & $54-31-9$ & Diuretic & Triclocarban & $101-20-2$ & Antibacterial & $\alpha$-endosulfan & 959-98-8 & Organochlorine pesticide \\
\hline Gemfibrozil & 25812-30-0 & Lipid regulator & Triclosan $^{\mathrm{b}}$ & $3380-34-5$ & Antibacterial & $\beta$-endosulfan & $33213-65-9$ & Organochlorine pesticide \\
\hline Glibenclamide & 10238-21-8 & Anti-diabetic medication & & & & $\gamma$-chlordane & 5103-74-2 & Organochlorine pesticide \\
\hline Hydrochlorotiazide & $58-93-5$ & Diuretic & & & & & & \\
\hline Ibuprofen ${ }^{\mathrm{b}}$ & $15687-27-1$ & Analgesic/anti-inflammatory & & & & & & \\
\hline Indomethacine & $53-86-1$ & Analgesic/anti-inflammatory & & & & & & \\
\hline Ivermectin & 71827-03-7 & Other antibiotics & & & & & & \\
\hline Ketoprofen & 22071-15-4 & Analgesic/anti-inflammatory & & & & & & \\
\hline Lincomycin & $154-21-2$ & Lincosamide (antibiotic) & & & & & & \\
\hline Mefenamic Acid & $61-68-7$ & Analgesic/anti-inflammatory & & & & & & \\
\hline Metoprolol & 51384-51-1 & $\beta$-blocker (antihypertensive) & & & & & & \\
\hline Methotrexate & $59-05-2$ & Other PhACs & & & & & & \\
\hline Metronidazole & $443-48-1$ & Nitroimidazol (antibiotic) & & & & & & \\
\hline Monensin & 17090-79-8 & Other antibiotics & & & & & & \\
\hline Nadolol & $42200-33-9$ & $\beta$-blocker (antihypertensive) & & & & & & \\
\hline Naproxen ${ }^{b}$ & 22204-53-1 & Analgesic/anti-inflammatory & & & & & & \\
\hline Nitrofurantoin & $67-20-9$ & Nitroimidazols & & & & & & \\
\hline Norfloxacin & 70458-96-7 & Quinolone (antibiotic) & & & & & & \\
\hline Novobiocin & $303-81-1$ & Aminocoumarin antibiotic & & & & & & \\
\hline Ofloxacin & 82419-36-1 & Quinolone (antibiotic) & & & & & & \\
\hline Ornidazole & $16773-42-5$ & Nitroimidazol (antibiotic) & & & & & & \\
\hline Oxacillin & $66-79-5$ & Penicillin (antibiotic) & & & & & & \\
\hline Oxytetracycline & $79-57-2$ & Tetracyclines & & & & & & \\
\hline Penicillin-G & $61-33-6$ & Penicillin (antibiotic) & & & & & & \\
\hline
\end{tabular}


Table 3. Cont

\begin{tabular}{|c|c|c|c|c|c|c|c|c|c|}
\hline \multicolumn{10}{|c|}{ Compounds } \\
\hline Pharmaceuticals & CAS & Use & OPFRs & CAS & Use & PAHs & CAS & PCBs & CAS \\
\hline Phenazone & $60-80-0$ & Phenazone type & TBP-N & $126-73-8$ & Flame retardant & Acenaphthene & $83-32-9$ & РСB28 & $7012-37-5$ \\
\hline Phenylbutazone & $50-33-9$ & Phenazone type & $\mathrm{TPP}^{\mathrm{b}}$ & $115-86-6$ & Flame retardant & Acenaphthylene & $208-96-8$ & PCB52 & $35693-99-3$ \\
\hline Pindolol & $13523-86-9$ & $\beta$-blocker (antihypertensive) & & & & Anthracene ${ }^{\text {a }}$ & $120-12-7$ & PCB101 & $37680-73-2$ \\
\hline Pravastatin & 81093-37-0 & Lipid regulator & & & & $\begin{array}{l}\text { Benzo }[b] \\
\text { fluoranthene }{ }^{a}\end{array}$ & $205-99-2$ & PCB138 & $35065-28-2$ \\
\hline Propanolol & $525-66-6$ & $\beta$-blocker (antihypertensive) & & & & $\begin{array}{l}\text { Benzo [k] } \\
\text { fluoranthene }{ }^{a}\end{array}$ & $207-08-9$ & PCB153 & $35065-27-1$ \\
\hline Ranitidine & $66357-35-5$ & $\begin{array}{l}\text { Histamine H2/receptor } \\
\text { antagonists }\end{array}$ & & & & $\begin{array}{l}\text { Benzo[a] } \\
\text { anthracene }\end{array}$ & $56-55-3$ & PCB180 & $35065-29-3$ \\
\hline Rifanpicim & $13292-46-1$ & Other antibiotics & & & & Benzo[a] pyrene ${ }^{a}$ & $50-32-8$ & & \\
\hline Roxithromycin & 80214-83-1 & Macrolide & & & & $\begin{array}{l}\text { Benzo[g,h,i] } \\
\text { perylene }^{\mathrm{a}}\end{array}$ & $191-24-2$ & & \\
\hline Salicylic Acid ${ }^{\mathrm{b}}$ & $69-72-7$ & Analgesic/anti-inflammatory & & & & Chrysene & $218-01-9$ & & \\
\hline Sparfloxacin & $110871-86-8$ & Quinolone (antibiotic) & & & & $\begin{array}{l}\text { Dibenzo[a,h] } \\
\text { anthracene }\end{array}$ & $53-70-3$ & & \\
\hline Spyramycin & $8025-81-8$ & Macrolide & & & & Fluoranthene $^{\mathrm{a}}$ & $206-44-0$ & & \\
\hline Sulfadiazine & $68-35-9$ & Sulfonamide (antibiotic) & & & & Fluorene & $86-73-7$ & & \\
\hline Sulfadimethoxine & $122-11-2$ & Sulfonamide (antibiotic) & & & & $\begin{array}{l}\text { Indeno }[1,2,3-C d] \\
\text { pyrene }^{\text {a }}\end{array}$ & $193-39-5$ & & \\
\hline Sulfaguanidine & $57-67-0$ & Sulfonamide (antibiotic) & & & & Phenanthrene & $85-01-8$ & & \\
\hline Sulfamethazine & $57-68-1$ & Sulfonamide (antibiotic) & & & & Pyrene & $129-00-0$ & & \\
\hline Sulfamethizole & $144-82-1$ & Sulfonamide (antibiotic) & & & & & & & \\
\hline Sulfamethoxazole & $723-46-6$ & Sulfonamide (antibiotic) & & & & & & & \\
\hline Sulfamethoxipyridazine & $80-35-3$ & Sulfonamide (antibiotic) & & & & & & & \\
\hline Sulfanilamide & $63-74-1$ & Sulfonamide (antibiotic) & & & & & & & \\
\hline Sulfathiazole & $72-14-0$ & Sulfonamide (antibiotic) & & & & & & & \\
\hline Sulfisoxazole & $127-69-5$ & Sulfonamide (antibiotic) & & & & & & & \\
\hline Tetracycline & $60-54-8$ & Tetracyclines & & & & & & & \\
\hline Tiamulin & $55297-95-5$ & Amphenicol & & & & & & & \\
\hline Timolol & $26839-75-8$ & $\beta$-blocker (antihypertensive) & & & & & & & \\
\hline Trimethoprim & 738-70-5 & Dihydrofolate (antibiotic) & & & & & & & \\
\hline Tylosin & $1401-69-0$ & Macrolide & & & & & & & \\
\hline
\end{tabular}




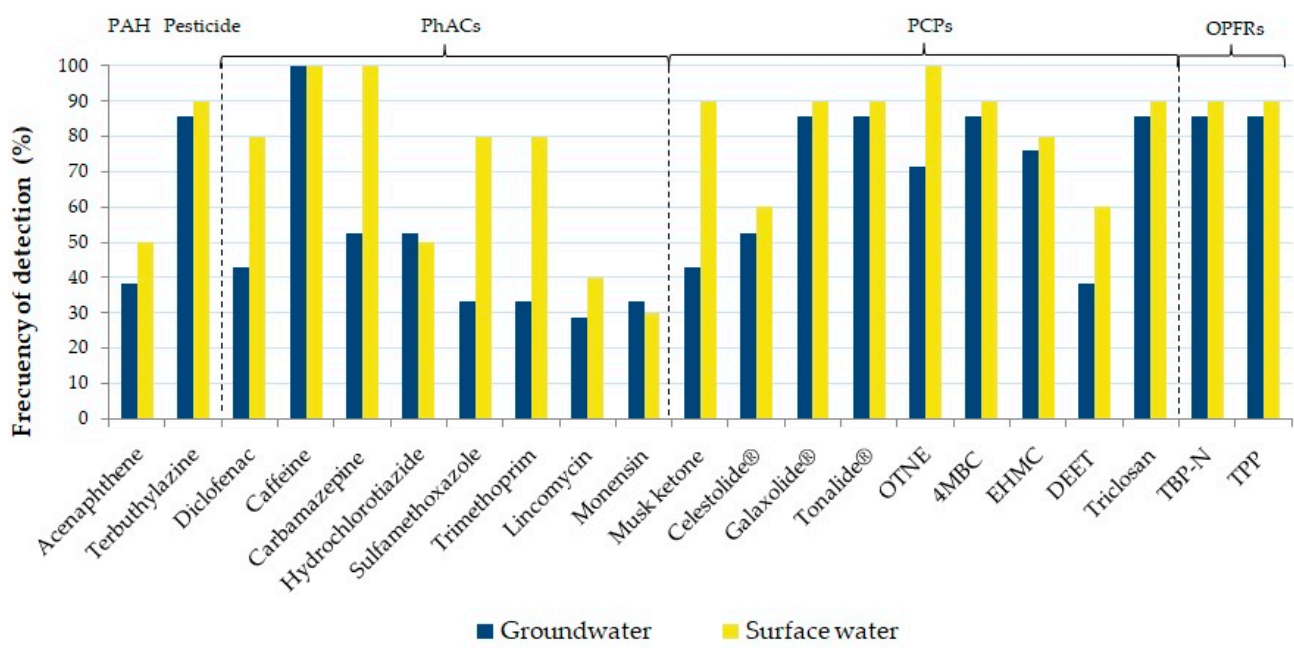

Figure 4. Compounds with a detection frequency in wells higher than $25 \%$ sorted by groups: polycyclic aromatic hydrocarbons (PAHs), pesticides, pharmaceutically active compounds (PhACs), personal care products (PCPs) and organophosphorus flame retardants (OPFRs).

Among the 63 detected compounds, antibiotics, the pesticide terbuthylazine, the PCP triclosan and the PAH acenaphthene were selected for discussion. The selection was based on results from previous studies, use of the compounds, frequency of detection, persistence in aquatic media and environmental risk.

\subsection{Antibiotics}

Most antibiotics were detected in the lower sub-basin (sector A; Figure 5). Among them, sulfamethoxazole was one of the most frequently detected. It is one of the sulfonamides found in the basin, which is a group of antibiotics commonly used in both veterinary and human medicine [30]. Sulfamethoxazole was detected in $50 \%, 22 \%$ and $25 \%$ of the groundwater samples of sectors A, B and C, respectively. In groundwater, the highest concentration was registered at point G26 (29.1 ng/L), whereas the highest values in surface waters where recorded in the river mouth, S27 (128 ng/L), and at the head of the Guadalteba River (S15: $56.2 \mathrm{ng} / \mathrm{L}$ ).

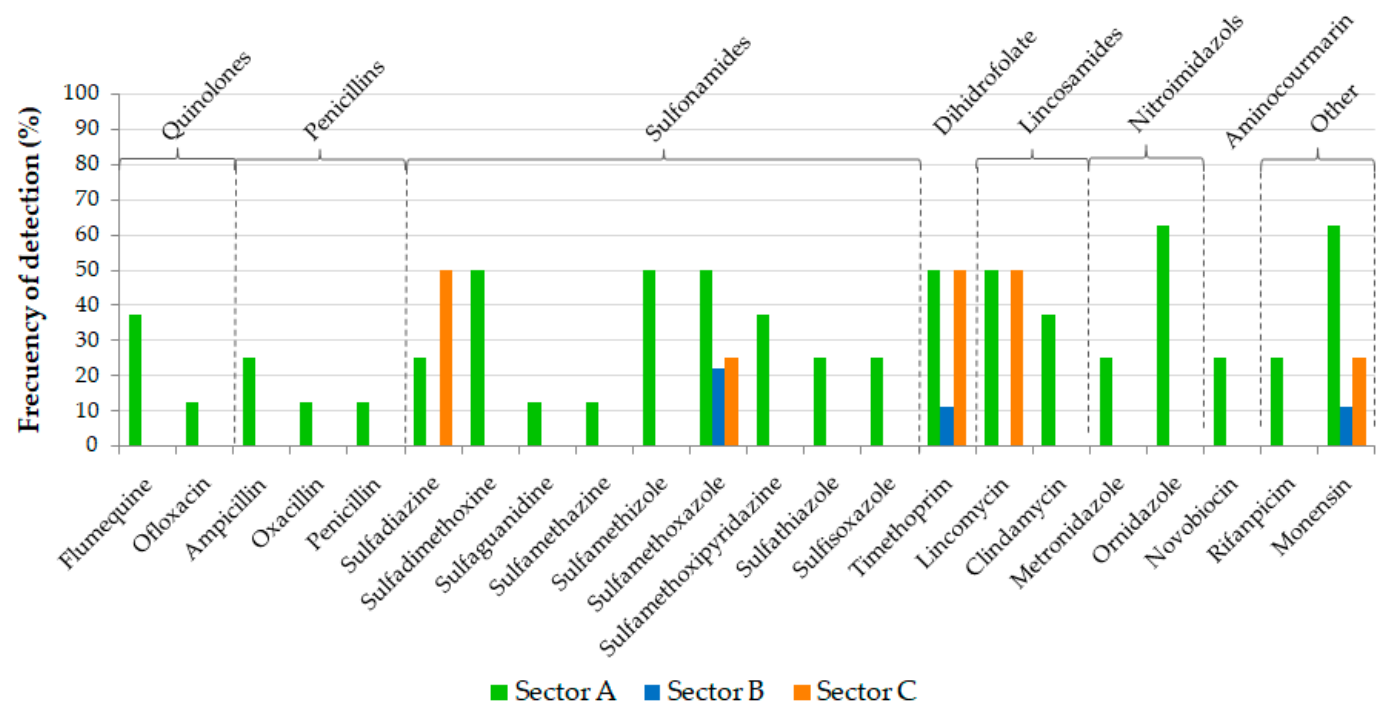

Figure 5. Frequency of detection of antibiotics in groundwater in sectors A, B and C.

In a study conducted with the aim of assessing the environmental factors on groundwater antibiotic occurrence, Menció and Mas-Pla [16] observed that antibiotic spatial distribution was statistically more 
related to groundwater properties than to parameters linked to potential sources, although solute transport parameters remained essential in order to fully explain antibiotic spatial distribution.

Sulfonamides are ampholitic compounds and sulfamethoxazole dissociation constants are $\mathrm{pK}_{\mathrm{a}, \text { acid }}$ $=1.6$ and $\mathrm{pK}_{\mathrm{a} \text {,base }}=5.7$ [30], which means that it is negatively charged under typical environmental $\mathrm{pH}$ conditions $(\mathrm{pH} \approx 7-9)$. The $\mathrm{pH}$ values recorded in groundwater across the basin ranged from 7.03 to 9.13. Thus, these compounds can migrate easily due to repulsion from negatively charged surfaces of clays and organic matter. Furthermore, they have a low $\log \mathrm{K}_{\mathrm{ow}}$ (-0.1 to 1.7 [31]), and therefore avoid hydrophobic sorption. Sulfamethoxazole has not been identified as either easily biodegradable or sensitive to photolysis under neutral and basic $\mathrm{pH}$ conditions [32]. Residues of sulfamethoxazole have also been found in other basins [33]. The persistence of this compound, along with its capability to avoid sorption, is potentially a reason for its wide distribution.

Despite the high frequency of detection in sector A, many potential point sources of antibiotics such as pig farms are mainly located in the northwestern part of the basin (sector C) whose waste waters, along with those from urban areas, enter the Venta River, the main stream in sector $C$ that flows into the dam system in the center of the basin. The antibiotics distribution in the target areas presumably responds to two factors: the hydrogeological dynamics (modified by hydraulic infrastructures) of the basin and the use of pig manure as an organic fertilizer in agriculture.

The three dams situated in the center of the basin collect the surface water from the upper basin (sectors B and C) to fulfill water demand. The Guadalhorce River in sector B is hydraulically connected with the porous aquifer, whereas the Venta River, sector $C$, recharges the aquifers which finally drain through a spring outflowing into the dam. The surface water is eventually released downstream (towards sector A) through irrigation channels and to the Guadalhorce riverbed. The effect of surface water flowing from dams into the aquifer of the lower sub-basin (sector A) is reflected in Figure 6. An enrichment of the water isotopic composition $\left(\delta^{2} \mathrm{H} / \delta^{18} \mathrm{O}\right)$ is observed in waters of sector $\mathrm{A}$ in comparison to waters from the upper basin.

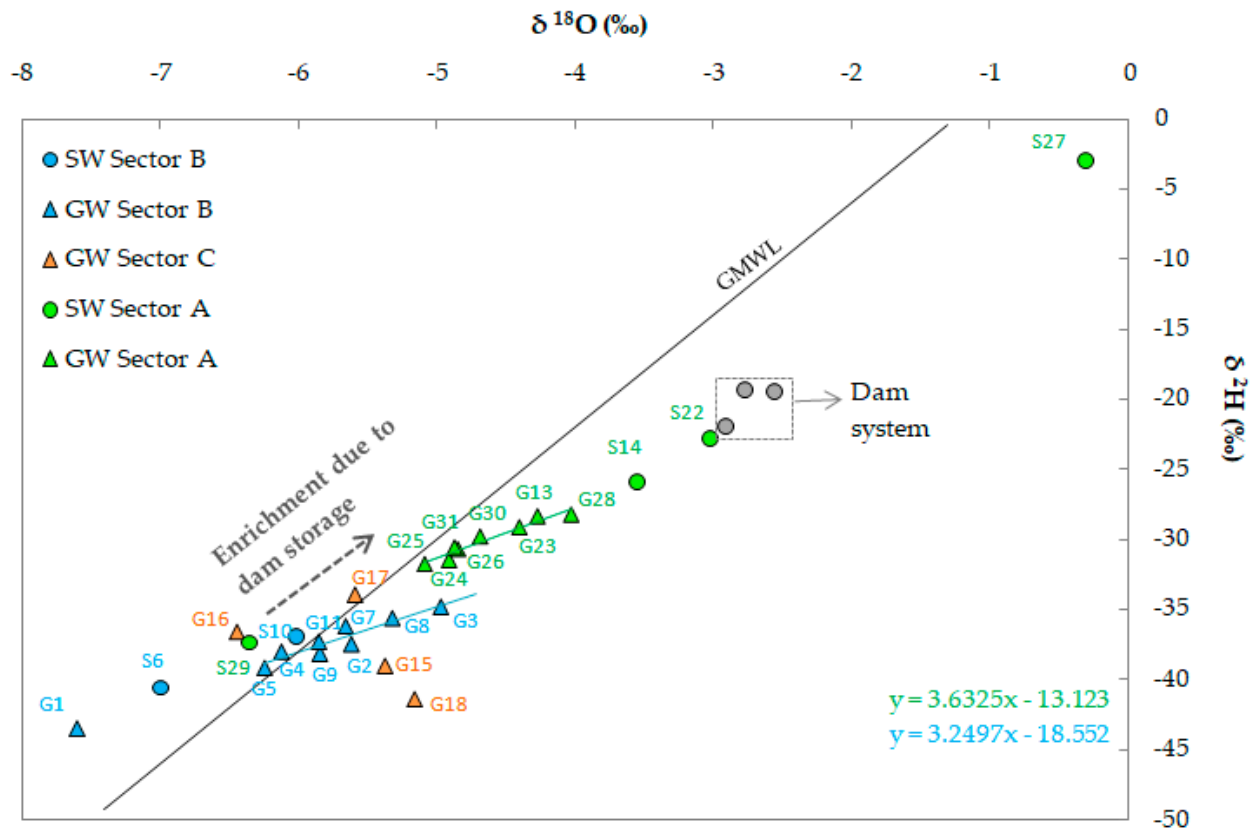

Figure 6. Relation of $\delta^{2} \mathrm{H}$ and $\delta^{18} \mathrm{O}(\%$ o) of water molecules in monitored surface waters (SWs) and groundwater (GW) in the Guadalhorce River basin.

Sampled groundwater with high concentrations of chloride showed higher values of $\delta^{18} \mathrm{O}(\% \mathrm{o})$ (Figure 7). Accordingly, water used for irrigation infiltrates into the aquifer and is evaporated and salinized, and then it is pumped back to the surface and further reused. 


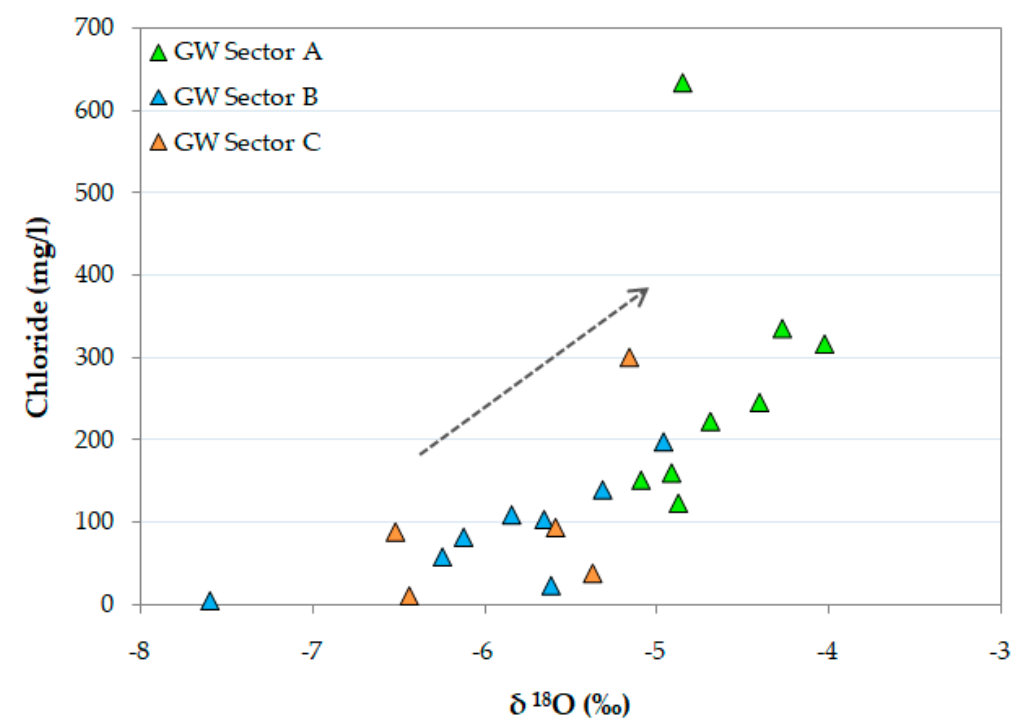

Figure 7. Relation of chloride $(\mathrm{mg} / \mathrm{L})$ and $\delta^{18} \mathrm{O}(\%)$ of monitored groundwater in the target areas $\mathrm{A}, \mathrm{B}$ and $\mathrm{C}$.

Since some antibiotics are found in the upper and the lower basin (sulfadiazine, sulfamethoxazole, timethoprim, lincomycin and monensin), and since they are more frequently detected in the lower sub-basin where they also present a higher concentration (Figure 8), it can be assumed that possible antibiotic sources exist in both sub-basins; however, these chemicals tend to accumulate in sector A. This tendency is probably a consequence of groundwater-surface water exchange processes and of the hydraulic infrastructure that conducts surface water from the upper basin towards the lower basin aquifer through the dams and through irrigation (Figure 6). Irrigation returns (Figure 7) also potentially contribute to pollutant accumulation.

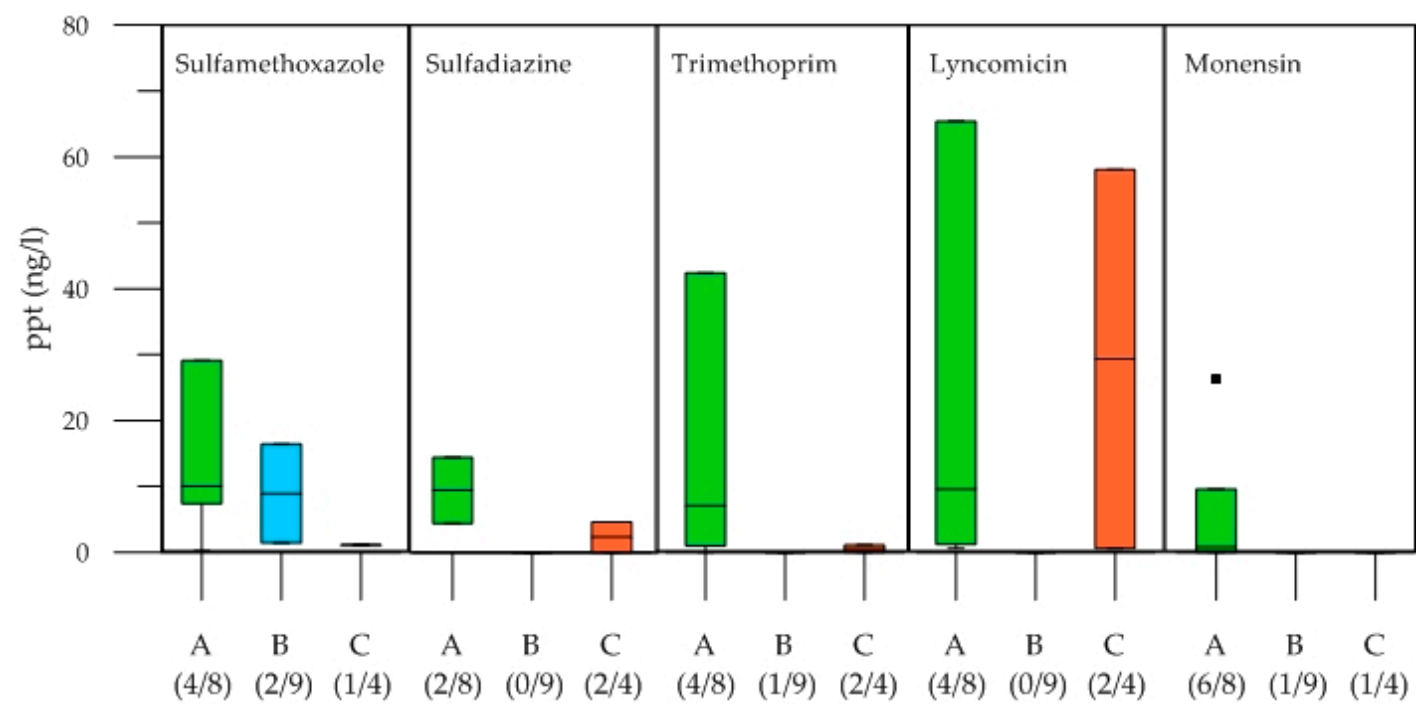

Figure 8. Box plots showing the concentration distribution of detected antibiotics sulfamethoxazole, sulfadiazine, trimethoprim, lyncomicin and monensin in groundwater in sectors A, B and C. Numbers in parentheses indicate the number of samples with detectable concentrations of compounds out of the total number of samples collected in the sector.

Pig manure used as fertilizer in agriculture is another potential antibiotics source. It is abundantly applied to crops during the spring and autumn in the northwestern area (sector C) and also in the lower part, along with chemical fertilizers for citrus crops [27]. Other potential sources include non-treated 
urban waste water discharges into the water courses all along the basin. Small urban areas (mainly in the lower part) are not connected to sewer systems [17].

\subsection{Pesticides. Terbuthylazine}

The broad spectrum herbicide terbuthylazine was present in $85 \%$ of the groundwater samples of the basin with a concentration ranging from $2.21 \mathrm{ng} / \mathrm{L}$ to $5.73 \mathrm{ng} / \mathrm{L}$. It was detected in $100 \%$ of groundwater samples in sector B, whereas in both sector A and C, it was found in $75 \%$ of the samples. It has already been identified as one of the most commonly found pesticides in the area [17]. Terbuthylazine is a very weak base $\left(\mathrm{pK}_{\mathrm{a}}=2\right)$. Thus, a non-ionic species exists over nearly the entire $\mathrm{pH}$ range and its sorption is dominated by hydrophobic partitioning to sorbent organic matter $\left(\log \mathrm{K}_{\mathrm{ow}}=3.4\right.$ [34]). In fact, its persistence has been partly attributed to the strong adsorption capacity on humic substances $[35,36]$. Consequently, transport via sediment and organic matter during water infiltration through an unsaturated zone is an important pathway towards groundwater [37].

In the Guadalhorce River basin, agriculture, with irrigated and rain-fed crops, is the anthropogenic activity affecting the largest area (more than 50\% of the total area $[27,38]$ ). Irrigated agriculture is concentrated in the alluvial lands of the lower basin (sector A; mainly citrus crops) and in the central zone of the upper basin, in sector B (mainly herbaceous crops like wheat, barley, legumes or tubers). There are also four golf courses near the coast (sector A) and one near the town of Antequera (sector B), which possibly use fertilizers and pesticides as well for lawn maintenance.

\subsection{Personal Care Products. Triclosan}

The antimicrobial triclosan was detected in $85 \%$ of the groundwater samples in the basin, being more frequently detected in sector B (100\%), and present in $75 \%$ of groundwater samples of sectors $\mathrm{A}$ and $\mathrm{C}$. Triclosan presents an important hydrophobic adsorption potential $\left(\log \mathrm{K}_{\mathrm{ow}}=4.76\right.$ [39]), allowing for an efficient removal in WWTPs as it absorbs onto the sewage sludge. Consequently, one the most important sources of triclosan in the environment is the use of sewage sludge (biosolids) from WWTPs as a fertilizer for crops [40].

Both the use of biosolids as fertilizers and discharge of non-treated wastewater are possible sources in the Gualdahorce River basin [17]. Wastewater discharge is a direct input into surface water courses. Groundwater is potentially reached through application of reclaimed sewage sludge on agricultural land, thus leading to a widespread presence of the pollutant in the basin.

\subsection{Polycyclic Aromatic Hydrocarbons. Acenaphthene}

Acenaphthene was the only PAH detected with a significant frequency (Figure 4). It was found in $38 \%$ of total groundwater samples of the basin. It is mainly present in the lower part of the basin (sector A), being detected in $87 \%$ of the samples collected in this area. Acenaphthene concentration ranged from $0.66 \mathrm{mg} / \mathrm{L}$ (G30) to $0.84 \mathrm{mg} / \mathrm{L}$ (G31).

Low molecular weight PAHs (with two to three benzene rings), such as acenaphthene, are normally released during petroleum processing, whereas high molecular weight PAHs are considered to originate from combustion [41,42]. Petroleum-related activities such as gas stations and gas and oil pipelines exist in the Guadalhorce River basin (Figure 9). The Arahal-Málaga oil pipeline and the Puente Genil-Málaga gas pipeline traverse the basin from NW-SE and N-S, respectively [17]. Furthermore, the Puerto Llano-Málaga oil pipeline, currently out of service, traverses the basin along the left side of the lower part of the river [43]. Leaching from one of these sources in sector A should not be disregarded. 


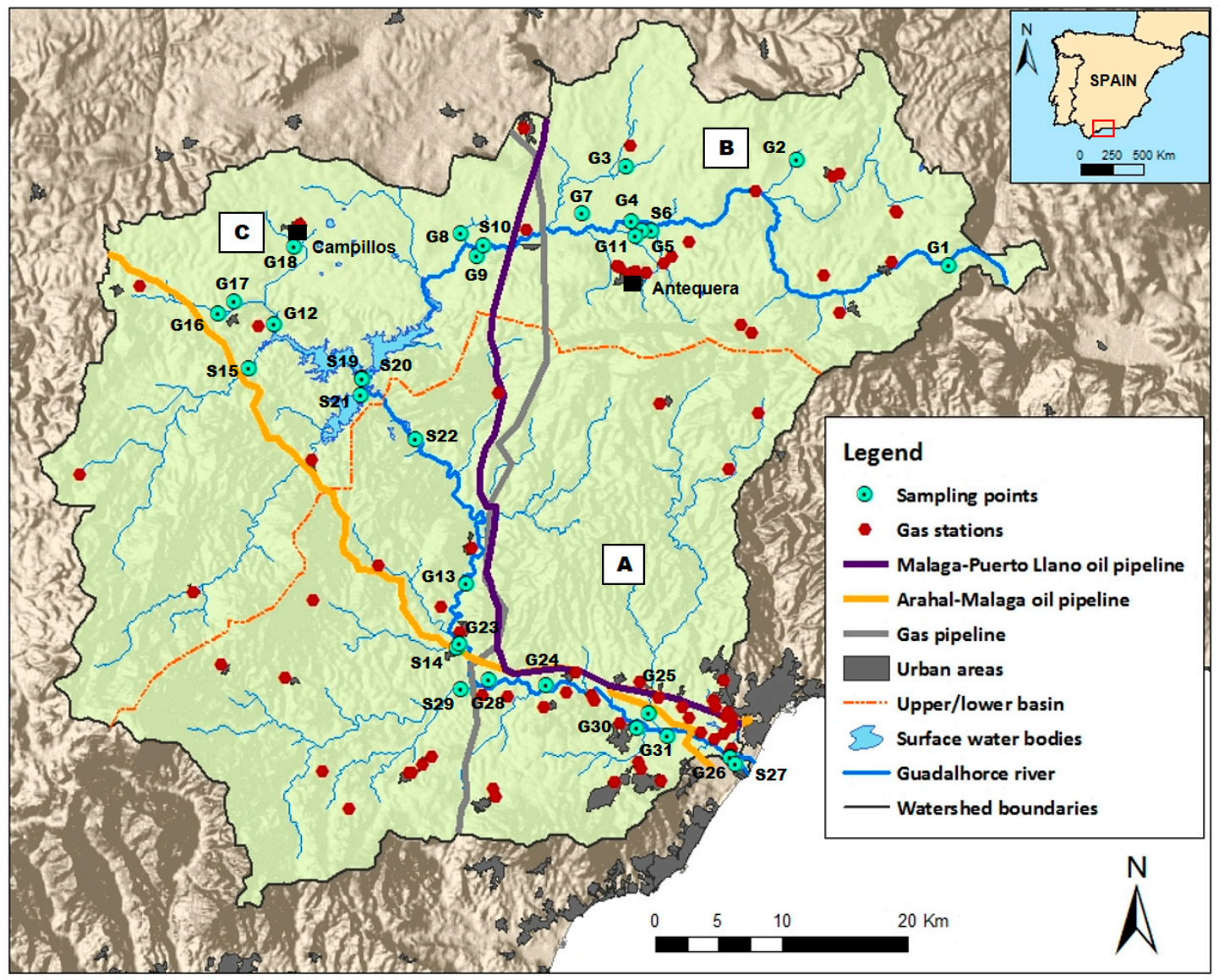

Figure 9. Hydrocarbon potential sources $[17,43]$ in Guadalhorce River basin and the three target areas (A, B and C) with the sampling points.

\section{Conclusions}

A first description of the occurrence and distribution of a wide range of regulated pollutants and contaminants of emerging concern in the Guadalhorce River basin has been drawn up. The results show the occurrence of 63 organic contaminants in surface and groundwater, 21 of which were found at least in $25 \%$ of the groundwater samples. Seventeen compounds were detected per groundwater sampling point, on average. The maximum number of contaminants was detected in a well situated near the river mouth (55 compounds), reflecting a strong influence on water quality from pollutant sources in this area, such as the discharge of treated and non-treated urban waste waters from urban areas and industrial sites.

Twenty-two different antibiotics have been found in water samples. Possible sources are waste water discharge into surface waters from urban areas and from intensive livestock production sites, which are especially numerous in the northwestern part of the basin. However, antibiotics spatial distribution does not show a correlation with the location of these potential sources. A possible tendency for antibiotics accumulation in the lower part of the basin is potentially attributed to surface-groundwater interactions and dynamics, which are modified by hydraulic infrastructures (dam system and irrigation channels).

On the contrary, the distribution of highly frequently detected triclosan and terbutylazine does not reflect a relation with water flow pathways, as they show a widespread distribution throughout the basin. The distribution difference with relation to antibiotics is attributed to the way these contaminants are potentially released into the environment, as well as their hydrophobicity. The herbicide terbuthylazine can derive from agriculture practices and the antimicrobial triclosan can derive from untreated urban wastewater discharges but also from the use of reclaimed sewage sludge as crop fertilizer. 
The sorption of these compounds is dominated by hydrophobic partitioning to sorbent organic matter. Presumably, they remain in the soil where they are added and slowly reach groundwater during water infiltration through the unsaturated zone.

Extremely high concentrations of acenaphthene (polyaromatic hydrocarbon) have been measured in water samples from the lower part of the river basin, ranging from $0.66 \mathrm{mg} / \mathrm{L}$ to $0.84 \mathrm{mg} / \mathrm{L}$. A possible leaching could be taking place from a petroleum-related activity or structure in this area.

It is known that full characterization and quantification in aquifer media and the fate of detected compounds is difficult and challenging and requires long-term monitoring to fully assess pollution exposure and to evaluate the response and correlation of analyzed organic chemicals to different hydrological factors, such as precipitation [12,44] and hydrochemical parameters [15,45].

Nevertheless, this is the first time an analysis and evaluation of such a great number of organic pollutants has been performed in this area. The obtained results yield important information for water resource management and provide a foundation for further research in the Guadalhorce River basin. Future monitoring practices and analyses can be optimized by focusing on selected contaminants and factors likely governing the distribution of the different pollutants. As observed, these factors can not only be the physico-chemical properties of the compounds, but also hydraulic infrastructures, which modify hydrogeological functioning of the basin and, thus, predetermine pollutant transportation and distribution.

Author Contributions: Conceptualization, I.V.-P., P.J.-G., L.C.; Methodology, I.V.-P., P.J.-G., C.C.-F., A.F.C.-G.; Sample collection, I.V.-P., P.J.-G., C.C.-F., A.F.C.-G.; Investigation and formal analysis, M.L., I.V.-P., L.C.-L., P.J.-G.; Writing—original draft, M.L.; Writing—review and editing, M.L., I.V.-P., L.C., P.J.-G., C.C.-F., A.F.C.-G.; Funding acquisition, I.V.-P.; Supervision, I.V.-P., P.J.-G., L.C.; Lab analysis, C.C.-F., I.V.-P. All authors have read and agreed to the published version of the manuscript.

Funding: This research received funding from the project "Study of emerging contaminants in four anthropized basins of South Spain (8.07/5.44.4757)".

Acknowledgments: This article is a contribution to the Research Group of the "Junta de Andalucía" (Group of Hydrogeology, RNM-308). The authors want to acknowledge the comments and suggestions of three anonymous reviewers and the Academic Editor for the improvement of the article.

Conflicts of Interest: The authors declare no conflict of interest.

\section{References}

1. Lapworth, D.J.; Baran, N.; Stuart, M.E.; Ward, R.S. Emerging organic contaminants in groundwater: A review of sources, fate and occurrence. Environ. Pollut. 2012, 163, 287-303. [CrossRef]

2. European Parliament and the Council of the European Union. Directive 2006/118/EC of the European Parliament and of the Council of 12 December 2006 on the protection of groundwater against pollution and deterioration. Off. J. Eur. Union 2006, L372/19. Available online: https://eur-lex.europa.eu/legal-content/EN/ TXT/?uri=celex\%3A32006L0118 (accessed on 20 June 2020).

3. European Commission. Commission Implementing Decision (EU) 2015/495 of 20 March 2015 establishing a watch list of substances for Union-wide monitoring in the field of wáter policy pursuant to Directive 2008/105/EC of the European Parliament and of the Council. Off. J. Eur. Union 2015, L78/40. Available online: https://eur-lex.europa.eu/legal-content/EN/TXT/PDF/?uri=CELEX:32015D0495\&from=PT (accessed on 20 June 2020).

4. Gago-Ferrero, P.; Díaz-Cruz, M.S.; Barceló, D. An overview of UV-absorbing compounds (organic UV filters) in aquatic biota. Anal. Bioanal. Chem. 2012, 404, 2597-2610. [CrossRef]

5. Lei, M.; Zhang, L.; Lei, J.; Zong, L.; Li, J.; Wu, Z.; Wang, Z. Overview of emerging contaminants and associated human health effects. Biomed. Res. Int. 2015, 2015, 404796. [CrossRef]

6. López-Pacheco, I.Y.; Silva-Núñez, A.; Salinas-Salazar, C.; Arévalo-Gallegos, A.; Lizarazo-Holguin, L.A.; Barceló, D.; Iqbal, H.M.N.; Parra-Saldívar, R. Anthropogenic contaminats of high concern: Existence in water resources and their adverse effects. Sci. Total Environ. 2019, 690, 1068-1088. [CrossRef]

7. Daughton, C.G.; Ternes, T.A. Pharmaceuticals and personal care products in the environment: Agents of subtle change? Environ. Health Perspect. 1999, 107, 907-938. [CrossRef] 
8. Daughton, C.G. Non-regulated water contaminants: Emerging research. Environ. Impact Assess. Rev. 2004, 24, 711-732. [CrossRef]

9. Teijon, G.; Candela, L.; Tamoh, K.; Molina-Díaz, A.; Fernández-Alba, A.R. Occurrence of emerging contaminants, priority substances (2008/105/CE) and heavy metals in treated wastewater and groundwater at Depurbaix facility (Barcelona, Spain). Sci. Total Environ. 2010, 408, 3584-3595. [CrossRef]

10. Estevez, E.; Cabrera, M.C.; Fernández-Vera, J.R.; Molina-Díaz, A.; Robles-Molina, J.; Palacios-Díaz, M.P. Monitoring priority substances, other organic contaminants and heavy metals in a volcanic aquifer from different sources and hydrological processes. Sci. Total Environ. 2016, 551-552, 186-196. [CrossRef]

11. Watanabe, N.; Bergamaschi, B.A.; Loftin, K.A.; Meyer, M.T.; Harter, T. Use and environmental occurrence of antibiotics in freestall dairy farms with manured forage fields. Environ. Sci. Technol. 2010, 44, 6591-6600. [CrossRef]

12. Corada-Fernández, C.; Candela, L.; Torres-Fuentes, N.; Pintado-Herrera, M.G.; Paniw, M.; González-Mazo, E. Effects of extreme rainfall events on the distribution of selected emerging contaminants in surface and groundwater: The Guadalete River basin (SW, Spain). Sci. Total Environ. 2017, 605-606, 770-783.

13. Gurr, C.J.; Reinhard, M. Harnessing natural attenuation of pharmaceuticals and hormones in rivers. Environ. Sci. Technol. 2006, 40, 2872-2876. [CrossRef]

14. Schaffer, M.; Licha, T. A framework for assessing the retardation of organic molecules in groundwater: Implications of the species distribution for the sorption-influenced transport. Sci. Total Environ. 2015, 524-525, 187-194. [CrossRef]

15. Massman, G.; Greskowiak, J.; Dünnbier, U.; Zuehlke, S.; Knappe, A.; Pekdeger, A. The impact of variable temperaturas on the redox conditions and the behaviour of pharmaceutical residues during artificial recharge. J. Hydrol. 2006, 328, 141-156. [CrossRef]

16. Menció, A.; Mas-Pla, J. Assessing the influence of environmental factor son groundwater antibiotic occurrence by means of variation partitioning. Water 2019, 11, 1495. [CrossRef]

17. Sánchez-García, D. Water Framework Directive 2000/60/CE Application in the Guadalhorce River Basin (Málaga). Ph.D. Thesis, University of Malaga, Malaga, Spain, 2010; Initial characterization. (In Spanish)

18. Galvez, R.; Orozco, M. Strain determinations using deformed Radiolaria. Malaguide Complex, Southern Spain. Acta Geol. Hisp. 1979, 14, 129-134.

19. Vadillo-Pérez, I.; Carrasco-Cantos, F.; Sánchez-García, D. Bajo Guadalhorce. In Atlas Hidrogeológico de la Provincia de Málaga, 1st ed.; Durán-Valsero, J.J., Andreo-Navarro, B., Eds.; IGME: Madrid, Spain; Diputación Provincial de Málaga: Málaga, Spain, 2007; Volume 2, pp. 180-184.

20. IGME. Hydrogeological Investigation of the Basins from Southern Spain (Western Sector); Technical Report $\mathrm{n}^{\circ} 5$, Aquifer System n³7 (Malaga Porous Aquifer); Spanish Geological Survey: Madrid, Spain, 1983; 130p. (In Spanish)

21. Linares, L.; López-Arechavala, G.; López-Geta, J.A.; Campos-Rubio, J.C. Geometrical definition of the Pliocene-Quaternary aquifers of the Guadalhorce valley (Málaga). In Proceedings of the VI Hydrogeology Symposium, Sevilla, Spain, 23-27 October 1995; pp. 435-447. (In Spanish)

22. Carrasco-Cantos, F. Contribution to the Knowledge of the Upper Basin of the Guadalhorce River: Physical Media. Ph.D. Thesis, University of Granada, Granada, Spain, 1986. (In Spainsh)

23. Carrasco-Cantos, F.; Sánchez-García, D.; Vadillo-Pérez, I. Sierra de Teba-Almargen-Campillos. In Atlas Hidrogeológico de la Provincia de Málaga, 1st ed.; Durán-Valsero, J.J., Andreo-Navarro, B., Eds.; IGME: Madrid, Spain; Diputación Provincial de Málaga: Málaga, Spain, 2007; Volume 2, pp. 95-100.

24. Baena-Nogueras, R.M.; Pintado-Herrera, M.G.; González-Mazo, E.; Lara-Martín, P.A. Determination of pharmaceuticals in coastal systems using solid phase extraction (SPE) followed by ultraperformance liquid chromatography-tandem mass spectrometry (UPLC-MS/MS). Curr. Anal. Chem. 2016, 12, 183-201. [CrossRef]

25. Pintado-Herrera, M.G.; González-Mazo, E.; Lara-Martín, P.A. Atmospheric pressure gas chromatography-time-of-flight-mass spectrometry (APGC-Tof-MS) for the determination of regulated and emerging contaminants in aqueous samples after stir bar sorptive extraction (SBSE). Anal. Chim. Acta 2014, 851, 1-13. [CrossRef]

26. Pintado-Herrera, M.G.; González-Mazo, E.; Lara-Martín, P.A. In-cell clean-up pressurized liquid extraction and gas chromatography - tandem mass spectrometry determination of hydrophobic persistent and emerging organic pollutants in coastal sediments. J. Chromatogr. A 2016, 1429, 107-118. [CrossRef] 
27. Urresti-Estala, B.; Vadillo-Pérez, I.; Jiménez-Gavilán, P.; Soler, A.; Sánchez-García, D.; Carrasco-Cantos, F. Application of stable isotopes $\left(\delta^{34} \mathrm{~S}-\mathrm{SO}_{4}, \delta^{18} \mathrm{O}-\mathrm{SO}_{4}, \delta^{15} \mathrm{~N}-\mathrm{NO}_{3}, \delta^{18} \mathrm{O}-\mathrm{NO}_{3}\right)$ to determine natural background and contamination sources in the Guadalhorce River Basin (southern Spain). Sci. Total Environ. 2015, 506-507, 46-57. [CrossRef]

28. European Parliament and the Council of the European Union. Directive 2013/39/EU of the European Parliament and of the Council of 12 August 2013 amending Directives 2000/60/EC and 2008/105/EC as regards priority substances in the field of water policy. Off. J. Eur. Union 2013, L226/1. Available online: https://eur-lex. europa.eu/LexUriServ/LexUriServ.do?uri=OJ:L:2013:226:0001:0017:EN:PDF (accessed on 20 September 2020).

29. Demeneix, B.; Slama, R. Endocrine disruptors: From scientific evidence to human health protection. Policy Department for Citizens' Rights and Constitutional Affairs. In Directorate General for Internal Policies of the Union; European Union: Brussel, Belgium, 2019. Available online: https://www.europarl.europa.eu/ thinktank/es/document.html?reference=IPOL_STU\%282019\%29608866 (accessed on 20 September 2020).

30. Boreen, A.L.; Arnold, W.A.; McNeill, K. Photochemical fate of sulfa drugs in the aquatic environment: Sulfa drugs containing five-membered heterocyclic groups. Environ. Sci. Technol. 2004, 38, 3933-3940. [CrossRef]

31. Avisar, D.; Lester, Y.; Ronen, D. Sulfamethoxazole contamination of a deep phreatic aquifer. Sci. Total. Environ. 2009, 407, 4278-4282. [CrossRef] [PubMed]

32. Baena-Nogueras, R.M.; González-Mazo, E.; Lara-Martín, P.A. Degradation kinetics of pharmaceuticals and personal care products in surface waters: Photolisis vs biodegradation. Sci. Total Environ. 2017, 590-591, 643-654. [CrossRef] [PubMed]

33. Candela, L.; Tamoh, K.; Vadillo, I.; Valdes-Abellan, J. Monitoring of selected pharmaceuticals over 3 years in a detrital aquifer during artificial groundwater recharge. Environ. Earth Sci. 2016, 75, 274. [CrossRef]

34. Tasca, A.L.; Puccini, M.; Fletcher, A. Terbuthylazine and desethylterbuthylazine: Recent occurrence, mobility and removal techniques. Chemosphere 2018, 202, 94-104. [CrossRef] [PubMed]

35. Dousset, S.; Mouvet, C.; Schiavon, M. Sorption of terbuthylazine and atrazine in relation to the physico-chemical properties of three soils. Chemosphere 1994, 28, 467-476. [CrossRef]

36. Navarro, S.; Vela, N.; Giménez, M.J.; Navarro, G. Persistence of four s-triazine herbicides in river, sea and groundwater samples exposed to sunlight and darkness under laboratory conditions. Sci. Total Environ. 2004, 329, 87-97. [CrossRef] [PubMed]

37. Kronvang, B.; Iversen, H.L.; Vejrup, K.; Mogensen, B.B.; Hansen, A.M.; Hansen, L.B. Pesticides in streams and subsurface drainage water within two arable catchments in Denmark. In Pesticide Application, Concentration, Transport and Fate; Danish Environmental Protection Agency: København, Denmark, 2003.

38. Junta de Andalucía (Andalusian Government). Cartographic Database SIOSE. Available online: http: //www.juntadeandalucia.es/medioambiente/site/rediam/menuitem.04dc44281e5d53cf8ca78ca731525ea0/ ?vgnextoid $=83539 \mathrm{c} 8 \mathrm{e} 47 \mathrm{~b} 3 \mathrm{c} 310 \mathrm{VgnVCM} 1000001325 \mathrm{e} 50 \mathrm{aRCRD \& vgnextchannel=}$ c2770219f560f210VgnVCM1000001325e50aRCRD\&vgnextfmt=rediam\&lr=lang_es (accessed on 20 September 2020).

39. Dhillon, G.S.; Kaur, S.; Pulicharla, R.; Brar, S.K.; Cledón, M.; Verma, M.; Surampalli, R.Y. Triclosan: Current status, occurrence, environmental risks and bioaccumulation potential. Int. J. Environ. Res. Public Health 2015, 12, 5657-5684. [CrossRef]

40. Chalew, T.E.A.; Halden, R.U. Environmental exposure of aquatic and terrestrial biota to triclosan and triclocarban. J. Am. Water Resourc. Assoc. 2009, 45, 4-13. [CrossRef]

41. Fernandes, M.B.; Sicre, M.A.; Boireau, A.; Tronczynski, J. Polyaromatic hydrocarbon (PAH) distributions in the Seine River and its estuary. Mar. Poll. Bull. 1997, 34, 857-867. [CrossRef]

42. Li, J.; Li, F.; Liu, Q. PAHs behavior in surface water and groundwater of the Yellow River estuary: Evidence from isotopes and hydrochemistry. Chemosphere 2017, 178, 143-153. [CrossRef]

43. Rodríguez-Paradinas, E.; Orozco-Vallejo, E. Málaga-Puerto Llano oil pipeline. Project and main pipeline construction. Revista de Obras Públicas 1967, 115, 97-111. Available online: http://ropdigital.ciccp.es/revista_ op/detalle_articulo.php?registro=16925\&anio=1967\&numero_revista=3022 (accessed on 20 September 2020). (In Spanish) 
44. Luque-Espinar, J.A.; Navas, N.; Chica-Olmo, M.; Cantarero-Malagón, S.; Chica-Rivas, L. Seasonal occurrence and distribution of a group of ECs in the water resources of Granada city metropolitana reas (South of Spain): Pollution of raw drinking water. J. Hydrol. 2015, 531, 612-625. [CrossRef]

45. You, L.; Nguyen, V.T.; Pal, A.; Chen, H.; He, Y.; Reinhard, M.; Gin, K.Y. Investigation of pharmaceuticals, personal care products and endocrine disrupting chemicals in a tropical urban catchment and the influence of environmental factors. Sci. Total. Environ. 2015, 536, 955-963. [CrossRef]

Publisher's Note: MDPI stays neutral with regard to jurisdictional claims in published maps and institutional affiliations.

(C) 2020 by the authors. Licensee MDPI, Basel, Switzerland. This article is an open access article distributed under the terms and conditions of the Creative Commons Attribution (CC BY) license (http://creativecommons.org/licenses/by/4.0/). 